account of the mobility of the kidneys an abdominal belt giving special support to the kidneys by means of rubber airpads was ordered. This belt was probably instrumental in causing the subsequent attack of intestinal obstruction, since the right air-pad would tend to displace the cxcum to the left.

For some time the patient went on very well and his general condition improved considerably. Further, he expressed himself as having derived considerable comfort from the belt. Rather more than three weeks after his visit to me, he was seized with colicky abdominal pain and complete stoppage of the bowels. As this had occurred before, no great notice was taken of it. The pain, however, got worse, and two days later he began to vomit. A medical man was called in on the fourth day of the attack and various aperients and numerous enemata were tried with negative result. The pain was now so severe that the patient could not lie down but spent his time sitting in a chair and walking about. I was asked to see him on Nov. 5th, six days after the onset of the attack. He was sitting on the edge of the bed and looked very ill, the face being drawn and pinched. After taking a mixture containing opium the previous day the vomiting had entirely ceased and the pain was much easier, but at intervals he still had severe spasms of colic. The tongue was brown but moist, the pulse was 110 , but strong and good, and the temperature was $98 \cdot 4 \circ \mathrm{F}$. Nothing whatever had been passed by the rectum for six days. The abdomen was moderately distended all over and on the left side, filling up the entire space between the costal margin and a horizontal line at the level of the umbilicus, was a tense rounded swelling which stood up strikingly above the general level of the abdomen. It was tympanitic on percussion and could not be moved. The maximum projection was several inches below the costal margin but the fingers could not be passed between the costal margin and the tumour. As it was late at night and the surroundings were unsuited for an abdominal section in the patient's own home, high turpentine enemata were ordered and instructions were left that if not better on the following day be should be moved into a nursing home for immediate operation. Delay seemed justified by the fact that both the patient and his medical adviser were satisfied that his general condition had improved since the preceding day. On the next day, as the obstruction remained unrelieved and the vomiting had returned, he was transferred to a nursing home and operation was carried out. The vomiting occurred at short intervals but only a small quantity of greenish fluid with a slight fæculent odour was brought up each time. The pulse-rate had increased to 126 .

Operation.-An incision was made through the right rectus muscle for the reason that it seemed possible that the enlarged right kidney might in some way have induced the obstruction. On opening the peritoneum a quantity of slightly blood-stained fluid escaped. Many distended coils of small intestine were found beneath the incision and on opening these out the mesentery was seen to be twisted and greatly stretched. It was much congested and showed many petechial hrmorrhages. The tumour in the left side was found to consist of the enormously distended cæcum and first part of the ascending colon. Just to the right of the middle line the ascending colon was crossed by the tightly stretched twisted mesentery which completely occluded the bowel. The cæcum was punctured when a great volume of gas and 60 ounces of liquid frees escaped. It was then found possible to lift the small intestines over to the left side of the abdomen and to bring the cæcum from behind the mesentery. While doing this the lumen of the small bowel near the duodenum was found occluded by a twist round the longitudinal axis of the bowel. This accounted for the fact that the vomit had been small in amount and practically non-fæculent. After operation a considerable quantity of brown fæculent matter was vomited. The third stage of the duodenum was freely moveable and directly continuous with the second stage. Finally, the abdominal wound was sewn up without drainage. To prevent a recurrence of the volvulus and to allow of an opening being easily made into the bowel in case of necessity a pouch of the huge flaccid creum was fixed by sutures to the anterior sheath of the rectus in the lower angle of the wound.

The patient stood the operation well. He vomited several times during the first 24 hours, the vomit consisting at first of strongly fæculent brown fluid and later of bile. Flatus was passed by the rectum after a few hours and the bowels acted naturally at the end of 48 hours. The pouch of cæcum was not opened and soon healed over, though some weakness of the scar and slight bulging on coughing persisted at that point. An uneventful recovery took place and at the end of a month he was able to return home feeling quite strong and better than he had been for two years. The urine still contained a moderate amount of pus and in all probability the right kidney will have to be explored in the near future.

This case is recorded on account of the rarity of the condition and the many interesting features it presented.

Birmingham.

\section{TWO CASES OF VOLVULUS ASSOCIATED WITH HERNIA.}

BY J. F. DOBSON, M.S. LOND., F.R.C.S. ENG., CLINICAT, LECTURER ON SURGERT, UNIVERSTTY OF LEEDS, ETC.

CASE 1.-A man, aged 43 years, was admitted to the Leeds General Infirmary on July 20th, 1907. He had suffered from a right inguinal hernia for some years which had not previously given him any trouble. 48 hours before admission he was seized with pain in the hernia, which became swollen and irreducible, and in the abdomen. The bowels had not been moved and vomiting had been constant. On admission he was in a state of collapse, with cold extremities and a rapid feeble pulse. There was a large, tender, irreducible inguinal hernia on the right side, which gave no impulse on coughing. The abdomen was distended and tender, but it was possible to make out a tense swelling in the right iliac region extending upwards towards the umbilicus of about the size of the foetal head. A diagnosis was made of a volvulus of the small intestine lying partly in the abdomen and partly in the hernial sac.

Under stovaine spinal anæsthesia an incision was made in the middle line below the umbilicus. There was a large quantity of blood-stained fluid, having a fæcal odour, in the abdominal cavity. Some 7 or 8 feet of the lower ileum were found to be twisted on its mesenteric attachment and lying partly in the hernial sac. The gut was of a deep plum colour with dark patches in places. The whole of the volvulus was excised and owing to the desperate condition of the patient Paul's tubes were inserted into the two ends ; the tubes were surrounded with ganze and the abdomen was closed as rapidly as possible. Death occurred 36 hours later.

CAsE 2-A man, aged 68 years, was admitted to the infirmary on June 13th, 1908. He had suffered from a right inguinal hernia for many years. On June 12th the hernia became suddenly painful and swollen and could not be reduced. This was followed by vomiting which continued up to the time of his admission.

The case was thought to be an ordinary strangulated hernia and, under chloroform, herniotomy was performed in the usual way. On opening the sac, which contained a quantity of fluid, coils of normal small intestine were found lying side by side with other coils which were distended, thickened, and congested. There was no constriction at the neck of the sac, and on tracing the distended loops of gut upwards they were found to be continuous with other similar loops within the abdomen; these were withdrawn and it was then found that there was a volvulus of the small intestine involving two or three feet, the gut being twisted through one complete turn. The volvulus was untwisted and the intestine was returned into the abdomen. There was a large chronic vaginal hydrocele on the same side, and permission having been previously obtained the testicle was removed and the inguinal canal completely closed. Recovery ensued without incident.

The first of these two cases is of great interest in that it was possible to recognise the condition before operation and so to make an abdominal incision instead of one over the hernia, which would have been quite inadequate. It so happened that a short time previously I had re-read a reprint of Mr. R. Lawford Knaggs's paper in the Annals of Surgery for April, 1900, and recognition of the condition was therefore an easy matter. Both cases were of the same type, part of the volvulus lying in the hernial sac and part in the abdomen; in the first case many feet (probably about 8 ) of intestine were affected, while in the second case the volvulus 
was comparatively small; on the one hand the gut was on the point of becoming gangrenous and on the other it was merely congested and distended.

It is possible that had I ventured to prolong the operation in the first case sufficiently to effect an anastomosis instead of inserting Paul's tubes the patient would have stood a better chance of life.

Leeds.

\section{A SIMPLE METHOD OF ESTIMATING THE AMOUNT OF SUGAR IN GLYCOSURIC URINE.}

BY T. J. WALKER, M.D. LoND., F.R.C.S. ENG., cONSULTING SURGEON TO THE PETERBOROUGH INFIRMARY AND DISPENSARY.

(With Chart compiled by W. Elborne, M.A., Publio Analyst, Peterborough.)

IN this communication $I$ propose to bring before the busy practitioner, who has little time for laboratory work, a simple method of using Fehling's solution which I have long employed and which enables me, with the help of the accompanying chart, to determine in from three to six minutes the quantity of sugar in the urine of a glycosuric patient. The method of adding the urine minim by minim to the copper solution when performing a quantitative analysis of diabetic urine is now generally recommended, and various modifications of burette apparatus are described, the principle of all being the same; perhaps the most rapid of these tests being that with Carwardine's apparatus. It is the simplicity of my method and the fact that it can be performed with the ordinary test tube, pipette, spirit lamp, and minim measure in a far shorter time which constitute its value to the busy medical man.

The test solution I use is Fehling's solution, which should be recently prepared. The fundamental fact on which all quantitative tests by this solution rests is that in order to reduce to the insoluble suboxide the oxide of copper which is contained in a definite amount of the solution a definite amount of sugar is required. This is usually thus stated in metrical figures: "10 cubic centimetres of the solution require 50 milligrammes of glucose " completely to discharge the blue colour of the cupric sulphate and to convert all the copper to the condition of the suboxide. This being the case, if we take a measured quantity of Fehling's solution and ascertain the exact amount of any urine required to effect this reduction we can by calculation determine the percentage of sugar present in that urine.

In his usual qualitative testing with Fehling's solution every medical man must have observed (especially in urines containing a large amount of sugar) that the copper suboxide is occasionally thrown down as a pure red (scarlet or crimson) deposit, instead of the usual yellow, drab, or orange precipitate which as a rule occurs in the ordinary method of qualitative testing. Many years since, when, I believe, the burette quantitative test had not been described, it occurred to me that if I could determine the conditions which led to this red precipitate I might utilise its occurrence as an index of the amount of sugar present. I therefore varied my methods of using the test until I found that by adding the urine drop by drop to boiling Fehling's solution, boiling for a few seconds as each drop of urine was added, I could (except with urines containing only a very low', percentage of sugar) obtain the result constantly and not occasionally. I next established that the appearance of the blood-red precipitate corresponded with the complete reduction of the whole of the copper oxide to the suboxide and the discharge of all blue colour from the solution. I then adopted a definite amount of Fehling's solution-haif a drachm -as being the easiest quantity to manipulate in an ordinary fair-sized test-tube, which with this small quantity could be well tilted to prevent the sudden formation of steam from exploding the solution over the mouth of the tube. By adding the urine minim by minim from a hand pipette ${ }^{1}$ and noting the number of minims re-

1 I always use a pipette manufactured for me by Down Bros. It has a rubber ball at the top (practically a large nipple) which has a smaller capacity than the bulb of the pipette. By compressing the bulb and allowing it to expand again the urine is drawn into the pipette and by allowing it to expand again the urine is drawn into the pipette and by gentle pressure of the thumb in quired to effect the complete reduction of the copper I could calculate with sufficient accuracy for clinical purposes the amount of sugar in the urine under examination. (Fig. 1.) The essential point in manipulating this test is the

FIG. 1.

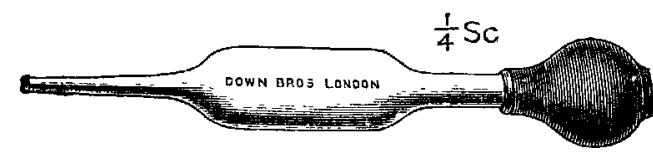

adding the urine drop by drop to the solution kept at boilingpoint until the point of saturation is reached, this being indicated by the formation of the dense crimson or scarlet precipitate and the discharge of all blue colour. If we use either more or less than the exact amount of urine necessary we do not obtain the same result, and even if we ascertain the precise amount of urine required and add it in bulk instead of drop by drop the blood-red precipitate is not formed, probably owing to the quantity of urine cooling down the solution. With urines containing not more than 5 or 6 grains in the ounce (about $1 \cdot 25$ per cent.) the precipi. tate, when saturation is complete, is rather a red orange than a pure red; it is therefore well to add another drop when the red tinge appears, and if with this extra drop the colour is not intensified we know that the saturation was complete before it was added and that it must not be reckoned in the number of minims required to effect complete reduction of the oxide.

To confirm the accuracy of my observations and conclusions I called in the assistance of a professional chemist, Mr. W. Elborne, borough analyst of Peterborough, and he explains the sudden formation of the red precipitate by what is called in chemistry the "mass action" of the elements concerned; the strong affinity between the constituents when they meet, under favourable conditions, in the exact proportions necessary for their entire absorption in the new chemical compound leads to the almos impulsive combination which gives us the pure red suboxide. Having satisfied himself by various corroborating experiments that the method of using Fehling's solution which I have described was reliable as a quantitative analysis sufficiently accurate for clinical purposes, Mr. Elborne suggested that its utility to the busy practitioner would be greatly enhanced if the value of the reaction between each minim of urine and 30 minims of Fehling's solution, as indicating the percentage of sugar, were shown by a curved line on a graduated chart, and he prepared such a chart which is here produced in the form we have finally adopted (Fig. 2); it shows at a glance, without any calculation, the number of grains per ounce and the percentage of sugar, represented by each minim of urine consumed in the test. The figures below the heary vertical lines on the chart indicate the number of minims of urine consumed in discharging the blue colour (and producing the pure red precipitate) from 30 minims of Fehling's solution. The figures opposite the heavy horizontal lines of the chart as well as those on the curved line crossing the chart indicate the percentage of sugar and the corresponding number (figures between brackets) of grains per fluid ounce. By percentage is understood "weight in volume"-i.e., grains per 100 minims.

Now, the reader who has followed me thus far will have noticed that I have occasionally spoken of drops, at other times of minims. The drop is not a uniform measure, and although for clinical purposes it may be used as though it were a minim it is not sufficiently accurate. But the drop is the measure that should for the sake of simplicity and rapidity be used (my pipette with rubber exhauster drops a minim of water, and I never consider it necessary to check it), and the difference, if there be any, between the drop and the minim can be determined in a few seconds by dropping from the pipette the same number of drops as have been used in the test into a minim measure, and, if the number of minims does not correspond with the number of drops, taking the number of minims as the figure which at the bottom of the chart indicates the point from which the eye should glance up to the point where the curve cuts the vertical line. For example, say 8 drops of urine have been added drop by drop to the boiling Fehling's solution before there forms the red rapidly falling precipitate leaving the fluid free from all blue colour, 8 drops of the same 